\title{
Effect of Streaming Motion of Baryons Relative to Dark Matter on the Formation of the First Stars
}

\section{Citation}

Stacy, Athena, Volker Bromm, and Abraham Loeb. 2011. "EFFECT OF STREAMING MOTION OF BARYONS RELATIVE TO DARK MATTER ON THE FORMATION OF THE FIRST STARS." The Astrophysical Journal 730 (1): L1. https://doi.org/10.1088/2041-8205/730/1/l1.

\section{Permanent link}

http://nrs.harvard.edu/urn-3:HUL.InstRepos:41412138

\section{Terms of Use}

This article was downloaded from Harvard University's DASH repository, and is made available under the terms and conditions applicable to Other Posted Material, as set forth at http:// nrs.harvard.edu/urn-3:HUL.InstRepos:dash.current.terms-of-use\#LAA

\section{Share Your Story}

The Harvard community has made this article openly available.

Please share how this access benefits you. Submit a story.

Accessibility 


\title{
EFFECT OF STREAMING MOTION OF BARYONS RELATIVE TO DARK MATTER ON THE FORMATION OF THE FIRST STARS
}

\author{
Athena Stacy ${ }^{1}$, Volker Bromm ${ }^{1}$, and Abraham Loeb ${ }^{2}$ \\ ${ }^{1}$ Department of Astronomy and Texas Cosmology Center, The University of Texas at Austin, TX 78712, USA; minerva@ astro.as.utexas.edu \\ 2 Astronomy Department, Harvard University, 60 Garden Street, Cambridge, MA 02138, USA \\ Received 2010 November 19; accepted 2011 January 31; published 2011 February 22
}

\begin{abstract}
We evaluate the effect of a supersonic relative velocity between the baryons and dark matter on the thermal and density evolution of the first gas clouds at $z \lesssim 50$. Through a series of cosmological simulations, initialized at $z_{\mathrm{i}}=100$ with a range of relative streaming velocities and minihalo formation redshifts, we find that the typical streaming velocities will have little effect on the gas evolution. Once the collapse begins, the subsequent evolution of the gas will be nearly indistinguishable from the case of no streaming, and star formation will still proceed in the same way, with no change in the characteristic Pop III stellar masses. Reionization is expected to be dominated by halo masses of $\gtrsim 10^{8} M_{\odot}$, for which the effect of streaming should be negligible.
\end{abstract}

Key words: cosmology: theory - early universe - galaxies: formation - stars: formation

Online-only material: color figures

\section{INTRODUCTION}

The formation of the first stars was a key event in the evolution of the early universe (e.g., Barkana \& Loeb 2001; Bromm \& Larson 2004; Ciardi \& Ferrara 2005; Glover 2005; Bromm et al. 2009; Loeb 2010). After the emission of the cosmic microwave background at $z \sim 1000$, the universe entered the "Dark Ages," the period when the distribution of matter was very uniform and no luminous objects had yet formed. During this time, cold dark matter (DM) density perturbations grew to make the halos inside of which the first stars formed at $z \lesssim 50$. These stars are believed to have formed within $M \sim 10^{6} M_{\odot}$ minihalos, where the infall of the baryons into the gravitational potential well of the DM-dominated minihalo heated the gas sufficiently to enable $\mathrm{H}_{2}$-driven cooling and fragmentation (e.g., Haiman et al. 1996; Tegmark et al. 1997; Yoshida et al. 2003).

The initial growth of the density fluctuations after recombination can be described using linear perturbation theory, which assumes that overdensities and velocity fields are small quantities. Similarly, cosmological simulations are initialized at high $z$ with small gas and DM peculiar velocities, determined through a combination of the $\Lambda \mathrm{CDM}$ model and the Zeldovich approximation (Zeldovich 1970). Recently, Tseliakhovich \& Hirata (2010) added a complicating aspect to this picture by showing that at high redshift, there is a supersonic relative velocity between the baryons and DM. Whereas prior to recombination, photons and baryons are coupled such that the baryonic sound speed is $\sim c / \sqrt{3}$, after recombination the sound speed drops to $\sim 6 \mathrm{~km} \mathrm{~s}^{-1}$. The root-mean-square relative velocity, on the other hand, is much higher, $30 \mathrm{~km} \mathrm{~s}^{-1}$. The relative velocities are dominated by modes on the comoving scale of $\sim 150 \mathrm{Mpc}$, the length scale of the sound horizon at recombination, and are coherent on smaller scales of a few Mpc.

Tseliakhovich \& Hirata (2010) examined how this effect alters the growth of DM structure, causing a small ( $\sim 10 \%)$ suppression of the matter power spectrum for modes with wavenumber $k \simeq 200 \mathrm{Mpc}^{-1}$. Using the Press-Schechter formalism they have also found a decrease in $M \sim 10^{6} M_{\odot}$ minihalos at high redshifts, $z=40$. They furthermore find that the relative velocity effect yields a scale-dependent bias of the first halos. Extending upon this, Dalal et al. (2010) analytically studied the impact of the relative velocity on baryonic objects, finding that the collapse fraction will be slightly reduced and that the large-scale clustering of $M \lesssim 10^{6} M_{\odot}$ minihalos will be modulated on scales of $\sim 100 \mathrm{Mpc}$. The same applies to any observable that traces minihalos, including the $21 \mathrm{~cm}$ absorption power spectrum. Tseliakhovich et al. (2010) find similar results in a more detailed analysis.

While these previous studies examined the large-scale effects of the relative velocity, its direct influence on the delay of collapse and the evolution of gas falling into a single minihalo has yet to be considered. Simulations are necessary to understand how the relative streaming affects the nonlinear regime and alters the processes involved in the collapse of minihalo gas. To this end, we perform a set of cosmological simulations which include these streaming motions. After the completion of this work, we became aware of an analogous paper by Maio et al. (2011). Similar to Maio et al. (2011), we find a delay of gas collapse in early low-mass $M \sim 10^{5}-10^{6} M_{\odot}$ minihalos, but conclude that for typical streaming velocities this delay will be negligible by $z \sim 10$. Our work is complementary to that of Maio et al. (2011) in that, while they are able to find a 1\%-20\% overall suppression of the first objects, our factor of $\sim 10$ greater mass resolution allows us to see the subsequent collapse of the gas to high densities, revealing that even with relative streaming motions the thermal evolution of primordial gas and subsequent Pop III star formation will be very similar to no-streaming cases following the initial collapse.

\section{NUMERICAL METHODOLOGY}

We carry out our investigation using GADGET, a widely tested three-dimensional smoothed particle hydrodynamics (SPH) code (Springel et al. 2001; Springel \& Hernquist 2002). Simulations are performed in a periodic box with size of $100 h^{-1} \mathrm{kpc}$ (comoving) and initialized at $z_{\mathrm{i}}=100$ with both $\mathrm{DM}$ and SPH gas particles. This is done in accordance with a $\Lambda$ CDM cosmology with $\Omega_{\Lambda}=0.7, \Omega_{\mathrm{m}}=0.3, \Omega_{\mathrm{B}}=0.04$, and $h=0.7$. We adopt $\sigma_{8}=0.9$ for the fiducial normalization of the power spectrum, and also examine the case of $\sigma_{8}=1.4$ in 
which structure formation is accelerated and the first minihalo collapses earlier. Each simulation box contains $128^{3} \mathrm{DM}$ particles and an equal number of SPH particles. The gas particles each have a mass $m_{\mathrm{SPH}}=8 M_{\odot}$, so that the mass resolution is $M_{\text {res }} \simeq 1.5 N_{\text {neigh }} m_{\mathrm{SPH}} \lesssim 400 M_{\odot}$, where $N_{\text {neigh }} \simeq 32$ is the typical number of particles in the SPH smoothing kernel (e.g., Bate \& Burkert 1997). This mass resolution allows us to follow the gas evolution to a maximum number density of $n_{\max }=10^{4} \mathrm{~cm}^{-3}$.

The chemistry, heating and cooling of the primordial gas is treated in a fashion very similar to previous studies (e.g., Bromm \& Loeb 2004; Yoshida et al. 2006). We follow the abundance evolution of $\mathrm{H}, \mathrm{H}^{+}, \mathrm{H}^{-}, \mathrm{H}_{2}, \mathrm{H}_{2}^{+}, \mathrm{He}, \mathrm{He}^{+}, \mathrm{He}^{++}, \mathrm{e}^{-}$, and the deuterium species $\mathrm{D}, \mathrm{D}^{+}, \mathrm{D}^{-}, \mathrm{HD}$, and $\mathrm{HD}^{+}$. We use the same chemical network as used in Greif et al. (2010) and include the same cooling terms.

We first perform both the "standard collapse" $\left(\sigma_{8}=0.9\right)$ and "early collapse" $\left(\sigma_{8}=1.4\right)$ initializations with no streaming velocity added. For each of these we also perform "moderate" and "fast" streaming cases in which we include an initial streaming velocity $v_{\mathrm{s}, \mathrm{i}}$ of $3 \mathrm{~km} \mathrm{~s}^{-1}$ and $10 \mathrm{~km} \mathrm{~s}^{-1}$, respectively. The "moderate" streaming case represents the predicted rootmean-square velocity (Tseliakhovich \& Hirata 2010), given that peculiar velocities have decreased as $(1+z)$ since recombination and thus have declined by a factor of 10 at the point our simulations are initialized. Our $v_{\mathrm{s}, \mathrm{i}}$ values therefore correspond to velocities of $30 \mathrm{~km} \mathrm{~s}^{-1}$ and $100 \mathrm{~km} \mathrm{~s}^{-1}$ at recombination, similar to the velocities chosen by Maio et al. (2011), 30 and $60 \mathrm{~km} \mathrm{~s}^{-1}$.

\section{RESULTS}

\subsection{Delay of Gas Collapse}

The main effect of the relative streaming cases is to delay the collapse of the baryons into the DM halos. In the standard case, the collapse redshifts are $z_{\mathrm{col}}=14.4,12.2$, and 6.6 for $v_{\mathrm{s}, \mathrm{i}}=0$, 3 , and $10 \mathrm{~km} \mathrm{~s}^{-1}\left(0,30\right.$, and $100 \mathrm{~km} \mathrm{~s}^{-1}$ at recombination). The streaming cases correspond to delays in collapse of $\sim 7 \times 10^{7}$ and $\sim 5 \times 10^{8}$ years, respectively. In the accelerated collapse case these values are $z_{\mathrm{col}}=23.6,21.3$, and 12.4, corresponding to delays of $\sim 2 \times 10^{7}$ and $\sim 2 \times 10^{8}$ years. Thus, this delay is noticeable only for high initial values of $v_{\mathrm{s}, \mathrm{i}} \gtrsim 3 \mathrm{~km} \mathrm{~s}^{-1}$, whereas at lower values the delay is small compared to the Hubble time.

We can understand the criterion for gas collapse in terms of the cosmological Jeans mass. In the usual no-streaming case, the slow infall of gas into the halos will first begin when the gravitational potential well of the minihalo, characterized by its virial velocity $V_{\text {vir }}$, is large enough to assemble the gas, which occurs when $V_{\mathrm{vir}}>c_{\mathrm{s}}$, where $c_{\mathrm{s}}=\sqrt{k_{B} T / \mu \mathrm{m}_{\mathrm{H}}}$ is the sound speed. Once this process begins, the sound speed $c_{\mathrm{s}}$ will be coupled to $V_{\mathrm{vir}}$ through adiabatic heating (see the top panels of Figure 1), and the density will scale with sound speed approximately as $c_{\mathrm{s}}^{3}$. In Figure 1, we determined the properties of the largest halo in our simulation using the HOP technique (Eisenstein \& Hut 1998) to find the DM particle in the region of highest DM density. Assuming this particle marks the center of the halo, the extent of the halo was determined by finding the surrounding spherical region in which the average DM density is $200 \rho_{c}$, where $\rho_{c}$ is the redshift-dependent critical density.

The bottom panels of Figure 1 illustrate that the adiabatic phase of evolution will continue until the virial mass of the minihalo is greater than the Jeans mass of the gas, $M_{\mathrm{vir}}>M_{\mathrm{J}}$.

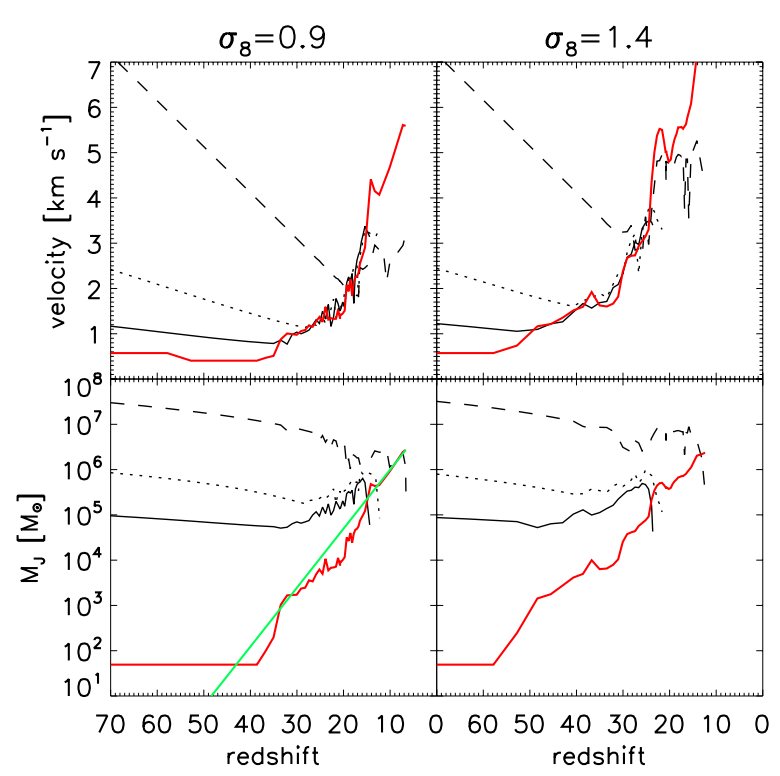

Figure 1. Top panels: effective velocity $v_{\mathrm{eff}}=\sqrt{c_{\mathrm{s}}^{2}+v_{\mathrm{s}}^{2}}$ of the gas (thin lines) and virial velocity $V_{\mathrm{vir}}$ of the simulated minihalo (thick red line). Top left: "standard collapse" case. Dashed line: $v_{\mathrm{s}, \mathrm{i}}=10 \mathrm{~km} \mathrm{~s}^{-1}$; dotted line: $v_{\mathrm{s}, \mathrm{i}}=$ $3 \mathrm{~km} \mathrm{~s}^{-1}$; solid black line: no-streaming case. At each redshift, $v_{\mathrm{s}}$ was found by taking an average over the entire gas within the simulation box. $c_{\mathrm{s}}$ refers to the average sound speed of all particles within the virial radius of the minihalo. Top right: early collapse case. Note that for the streaming cases the redshift at which $v_{\text {eff }}$ first falls below $v_{\text {vir }}$ matches well with the point where the gas thermal evolution first follows that of $v_{\text {vir }}$. Bottom panels: evolution of the Jeans mass $M_{\mathrm{J}}$ with redshift, evaluated using $v_{\text {eff }}$ in the role of the effective sound speed. Notation is the same as in the upper panels. Red line now shows virial mass $M_{\mathrm{vir}}$ of the minihalo. Green line is an exponential fit to the growth of the "standard collapse" case minihalo. Gas collapse occurs quickly after $M_{\mathrm{J}}$ drops below $M_{\text {vir }}$. Note that the enhancement of $v_{\text {eff }}$ due to the streaming velocity effectively increases $M_{\mathrm{J}}$, causing the gas collapse to be delayed until $M_{\mathrm{vir}}$ can further grow. This alters the final gas collapse redshifts of each case $\left(z_{\mathrm{col}}=14.4,12.2\right.$, and 6.6 for the no streaming, moderate streaming, and fast streaming cases given "standard collapse"; $z_{\text {col }}=23.6,21.3$, and 12.4 for "early collapse").

(A color version of this figure is available in the online journal.)

For the no-streaming case, we calculate $M_{\mathrm{J}}$ as

$$
M_{\mathrm{J}}=\left(\frac{\pi}{6}\right) \frac{c_{\mathrm{s}}^{3}}{G^{3 / 2} \rho^{1 / 2}} .
$$

Once the halo gains sufficient mass, and also provided that the $\mathrm{H}_{2}$-driven cooling time $t_{\text {cool }}$ of the gas is shorter than its freefall time $t_{\mathrm{ff}}$, the Jeans and cooling criteria will be satisfied and the gas will begin the next phase of rapid collapse to higher densities, quickly reaching $n_{\max }=10^{4} \mathrm{~cm}^{-3}$.

The cause for the delay in collapse of the streaming cases lies in the enhanced effective velocity of the gas,

$$
v_{\mathrm{eff}}=\sqrt{c_{\mathrm{s}}^{2}+v_{\mathrm{s}}^{2}}
$$

where the streaming velocity decreases with redshift as $v_{\mathrm{s}}(z)=$ $v_{\mathrm{s}, \mathrm{i}} /(1+z)$. As shown in the top panels of Figure 1, this delays the point at which the gas will begin falling into the halo. To accommodate the cases with streaming, we replace $c_{\mathrm{s}}$ in Equation (1) with $v_{\text {eff }}$, and the resulting increase of $M_{\mathrm{J}}$ is shown in the bottom panels of Figure 1.

For any given collapse redshift, a larger $M_{\mathrm{vir}}$ is therefore required to trigger the collapse of streaming gas compared with the non-streaming case. In Figure 2, we estimate for different redshifts the minimum halo mass into which gas with various initial streaming velocities can collapse. We arrive at 


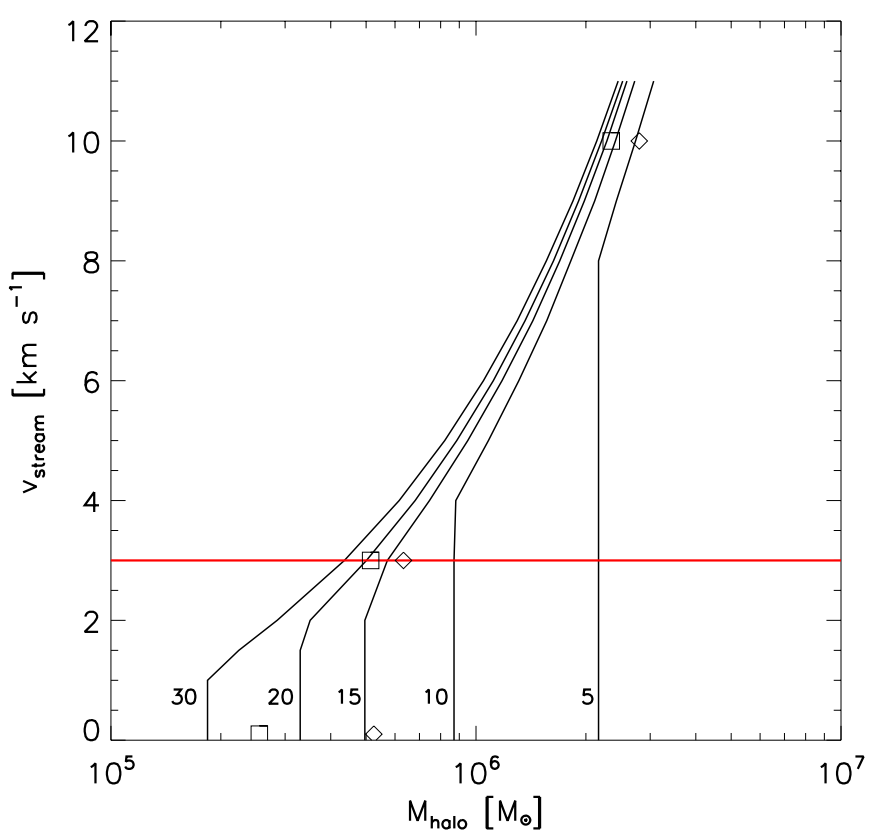

Figure 2. Effect of relative streaming on the minimum halo mass into which primordial gas can collapse. Each line represents the necessary halo masses for baryon collapse at a different redshift, marked in the plot. The diamonds represent the final halo masses found in "standard collapse" simulations $\left(z_{\mathrm{col}}=14\right.$ for no streaming $)$, and the squares represent masses from the "early collapse" simulations $\left(z_{\mathrm{col}}=24\right.$ for no streaming). Note that the halo mass does not noticeably increase unless the initial streaming velocities are very high $(\gtrsim 3$ $\mathrm{km} \mathrm{s}^{-1}$ ). Also note that halos collapsing at high redshift are more affected by relative streaming, as the physical streaming velocities are higher at these early times.

(A color version of this figure is available in the online journal.)

these estimates using the following simple prescription. We first determine for a range of redshifts the minihalo mass corresponding to a virial temperature of $1500 \mathrm{~K}$, which serves as the minimum mass for collapse and cooling given no streaming. We fit a typical halo growth history using $M_{\mathrm{vir}}(z)=M_{0} e^{\alpha z}$ (the green line shown in Figure 1), with $M_{0}=2 \times 10^{7} M_{\odot}$ and $\alpha$ ranging from -0.2 to -0.5 . We vary $\alpha$ depending on the nostreaming case minihalo mass and the desired collapse redshift. For every given collapse redshift and streaming velocity, we then determine the redshift $z_{\text {eq }}$, where $V_{\text {vir }}(z)=v_{\text {eff }}(z)$. We assume that $z_{\text {eq }}$ is the point where the gas switches from having properties of the intergalactic medium (IGM) to properties determined by the halo. Thus, for $z>z_{\text {eq }}$ the sound speed roughly follows that of the IGM, $c_{\mathrm{s}, \mathrm{IGM}}$. Therefore, $z_{\mathrm{eq}}$ can be found by considering

$$
V_{\mathrm{vir}}(z)=\sqrt{G M_{\mathrm{vir}}(z) / R_{\mathrm{vir}}(z)}=\sqrt{c_{\mathrm{s}, \mathrm{IGM}}(z)^{2}+v_{\mathrm{s}}(z)^{2}}
$$

where

$$
R_{\mathrm{vir}}(z) \simeq 210\left(\frac{M_{\mathrm{vir}}}{10^{6} M_{\odot}}\right)^{1 / 3}\left(\frac{1+z}{10}\right)^{-1} f(z) \mathrm{pc},
$$

and $f(z)$ is a factor of order unity with a mild dependence on redshift (Barkana \& Loeb 2001). At $z=z_{\text {eq }}$ the effective gas velocity is thus $v_{\mathrm{eq}}=V_{\mathrm{vir}}\left(z_{\mathrm{eq}}\right)$. After this point the thermal energy of the halo gas dominates over the energy of streaming motion, and its sound speed can be described by the halo virial velocity thereafter.

Furthermore, $z_{\text {eq }}$ marks the last time that the gas density within $R_{\mathrm{vir}}$ is still that of the IGM. The density of halo gas when it first couples to the DM is then

$$
\rho_{\mathrm{eq}}=\rho_{\mathrm{IGM}}\left(z_{\mathrm{eq}}\right) \simeq 2 \times 10^{-29} \Omega_{\mathrm{m}} h^{2}\left(1+z_{\mathrm{eq}}\right)^{3} \mathrm{~g} \mathrm{~cm}^{-3} .
$$

Note that, because $z_{\text {eq }}$ is lower for higher values of $v_{\mathrm{s}, \mathrm{i}}, \rho_{\mathrm{eq}}$ correspondingly decreases.

Finally, as the gas infall into the halo continues for $z<z_{\mathrm{eq}}$, we estimate its average density to be

$$
\rho(z) \simeq \rho_{\mathrm{eq}}\left(\frac{V_{\mathrm{vir}}(z)}{v_{\mathrm{eq}}}\right)^{3}
$$

The above equation describes how the gas density will adiabatically evolve with thermal energy as it collapses (e.g., Tegmark et al. 1997). Recall that $v_{\text {eq }}$ is the effective sound speed when it first begins falling into the halo, and that adiabatic evolution implies $T \propto \rho^{\gamma-1}=\rho^{2 / 3}$ for an atomic gas with $\gamma=5 / 3$. Using $c_{\mathrm{s}} \propto T^{1 / 2}$ results in $\rho \propto c_{\mathrm{s}}^{3}$. Finally, we replace $c_{\mathrm{s}}$ with the virial velocity of the halo to arrive at the approximation in Equation (6). The density will increase in this way until the gas virializes and reaches a maximum of $200 \rho_{\mathrm{IGM}}(z)$. Inserting the applicable values for $\rho$ and $V_{\text {vir }}$ at the given collapse redshift, $z_{\mathrm{col}}$, we arrive at $M_{\mathrm{J}}\left(z_{\mathrm{col}}\right)$.

This model well reproduces the masses and collapse redshifts found in the simulations (symbols in Figure 2). As the streaming velocities increase, the gas density during initial infall decreases, thereby lowering the typical gas density in the halo and raising the minimum mass that will satisfy $M_{\mathrm{vir}}>M_{\mathrm{J}}$. For the average $3 \mathrm{~km} \mathrm{~s}^{-1}$ streaming velocities, this minimum mass $M_{\text {halo }}$ will approximately double for $z_{\mathrm{col}}=30$, but will almost stay the same by $z_{\mathrm{col}}=10$.

\subsection{Thermal Evolution and Star Formation}

Figure 3 compares the thermal evolution for the "standard" and "early" collapse cases given no streaming with the corresponding rapid streaming cases $\left(v_{\mathrm{s}, \mathrm{i}}=10 \mathrm{~km} \mathrm{~s}^{-1}\right)$. For the no streaming cases, the gas follows the canonical evolution of adiabatic heating as the IGM gas gradually becomes incorporated into the growing minihalo. This gas heats to the virial temperature $(\sim 1000 \mathrm{~K})$ of the minihalo until the $\mathrm{H}_{2}$ fraction grows sufficiently high to allow the gas to cool to a minimum of $\simeq 200 \mathrm{~K}$. As the gas temperature drops, its density grows to approximately $10^{4} \mathrm{~cm}^{-3}$ (see Bromm et al. 2002; Yoshida et al. 2006). At this density the gas has reached the "loitering phase," and this is the reservoir of gas from which Pop III stars will form.

For the $v_{\mathrm{s}, \mathrm{i}}=10 \mathrm{~km} \mathrm{~s}^{-1}$ case, this evolution shows only minor differences from that described above. The initial heating of the low-density gas occurs more quickly than the purely adiabatic rate, and the streaming velocity acts as a heating term for the low-density gas. However, once the gas gains sufficiently high temperature and $\mathrm{H}_{2}$ fraction, the gas cools and condenses to approximately $200 \mathrm{~K}$ and $10^{4} \mathrm{~cm}^{-3}$, just as in the canonical case, though the minimum temperature is slightly lowered for the streaming cases. Subsequent star formation is therefore not suppressed, and should occur in the same way as it would in the no streaming case. Note also that Figure 3 represents significantly higher streaming velocities than typically expected. For the more representative $v_{\mathrm{s}, \mathrm{i}}=3 \mathrm{~km} \mathrm{~s}^{-1}$ cases, the thermal evolution shows almost no difference from those with no streaming. This further strengthens the argument that relative streaming between baryons and DM will do little to modify Pop III star formation.

Figure 4 further elucidates the effect of relative streaming on gas collapse and star formation. The delay of gas collapse 

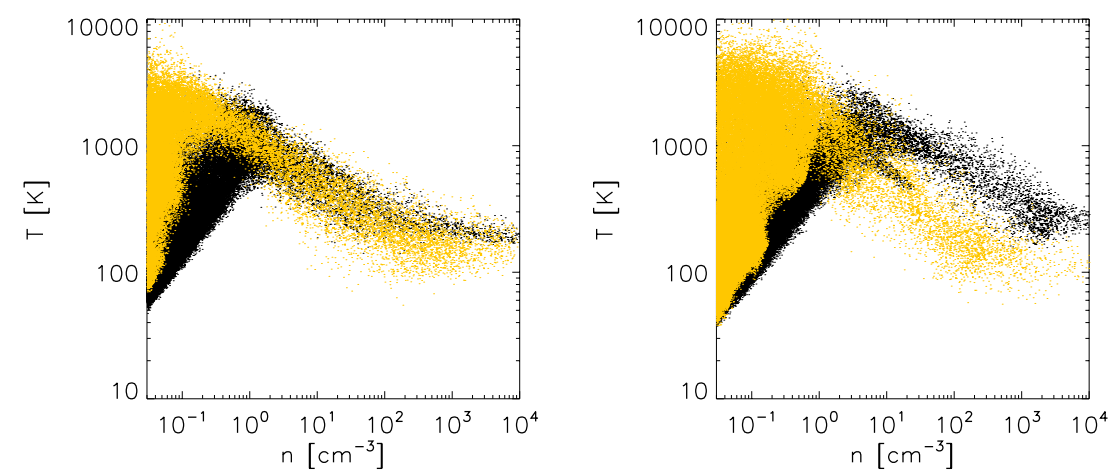

Figure 3. Evolution of temperature of the gas as density grows for both "standard" and "early" collapse cases. Black dots: no-streaming case. Yellow dots: $v_{\mathrm{s}, \mathrm{i}}=10$ $\mathrm{km} \mathrm{s}^{-1}$ case. Left: standard case is shown at $z=14.4$, while the $v_{\mathrm{s}, \mathrm{i}}$ case is shown at $z=6.6$. Right: early collapse case is shown at $z=23.6$ for no streaming, and $z=12.4$ for $v_{\mathrm{s}, \mathrm{i}}=10 \mathrm{~km} \mathrm{~s}^{-1}$. There is almost no difference between the $v_{\mathrm{s}, \mathrm{i}}=3 \mathrm{~km} \mathrm{~s}^{-1}$ (not shown) and no-streaming cases.

(A color version of this figure is available in the online journal.)

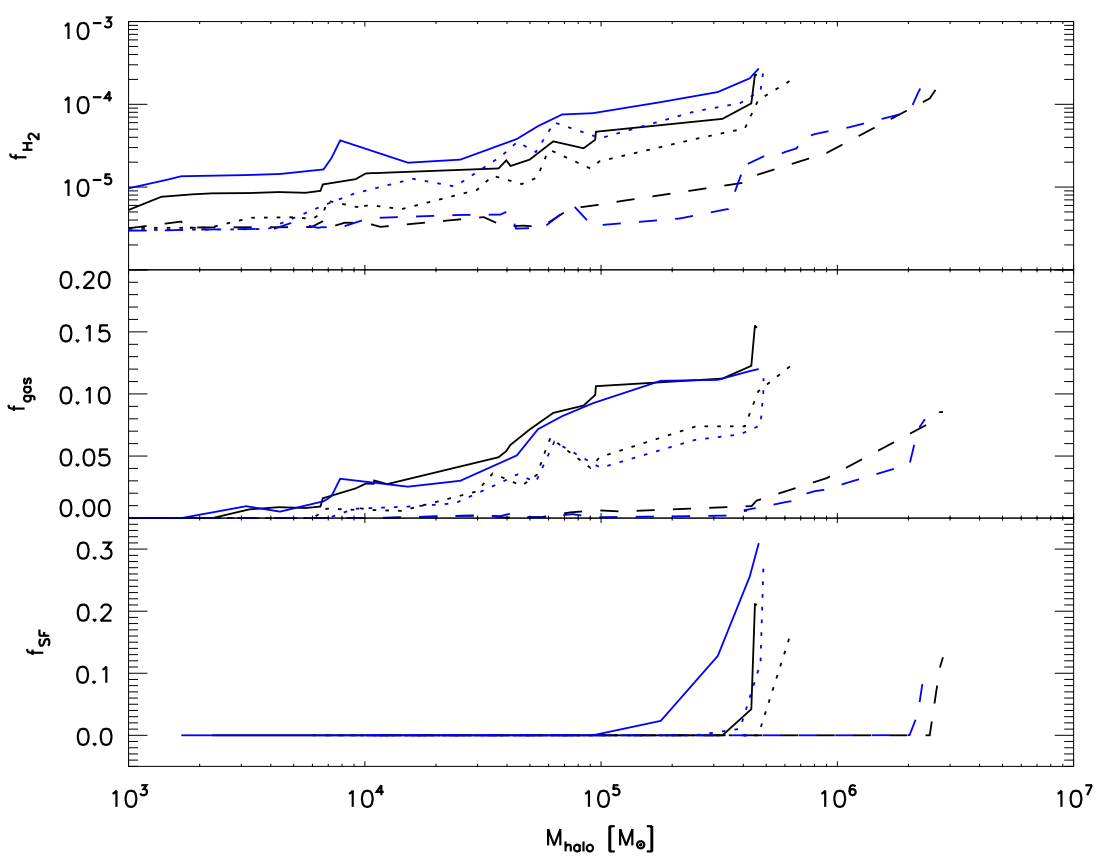

Figure 4. Evolution of gas properties with halo mass. Dashed lines: $v_{\mathrm{s}, \mathrm{i}}=10 \mathrm{~km} \mathrm{~s}^{-1}$; dotted lines: $v_{\mathrm{s}, \mathrm{i}}=3 \mathrm{~km} \mathrm{~s}^{-1}$; solid lines: no streaming. Black represents the "standard collapse" set of simulations, while blue represents the "early collapse" set. Top panel: average $\mathrm{H}_{2}$ fraction of the minihalo gas. Middle panel: gas fraction $f_{\text {gas }}$ of the halos. Bottom panel: fraction of minihalo gas that is star forming (i.e., $n>1 \mathrm{~cm}^{-3}$ ). The reduced gas fraction and the delay of star formation for high streaming velocities are evident in the bottom panels. However, the $\mathrm{H}_{2}$ fraction converges to $\gtrsim 10^{-4}$ in each case, allowing for the thermal evolution of the highest density gas to be relatively unchanged even for the highest streaming velocities.

(A color version of this figure is available in the online journal.)

until the minihalos have reached higher masses is evident in the bottom panel, which shows the fraction $f_{\mathrm{SF}}$ of the minihalo gas that is dense and star forming, defined as the gas that exceeds densities of $1 \mathrm{~cm}^{-3}$. There is no star-forming gas in $<10^{6} M_{\odot}$ halos for the highest streaming velocities. The gas fraction $f_{\text {gas }}$, calculated as the gas mass in the halo over its total mass, is reduced by up to a factor of $\sim 1.2$ for $v_{\mathrm{s}, \mathrm{i}}=3 \mathrm{~km} \mathrm{~s}^{-1}$ and a factor of $\sim 1.8$ for $v_{\mathrm{s}, \mathrm{i}}=10 \mathrm{~km} \mathrm{~s}^{-1}$, even after the gas has reached high densities. However, once gas collapse has occurred, the average $\mathrm{H}_{2}$ fraction $f_{\mathrm{H}_{2}}$ is very similar in all cases, as is the subsequent thermal evolution (Figure 3).

\section{DISCUSSION}

Our series of simulations show that Pop III star formation will be essentially the same in cosmologies with relative streaming motions between gas and DM, even in regions with streaming velocities much higher than average $\left(v_{\mathrm{s}, \mathrm{i}} \gtrsim 3 \mathrm{~km} \mathrm{~s}^{-1}\right)$. However, these regions of fast streaming will experience a modest delay in the collapse redshift at which Pop III stars will first form, while in regions of typical streaming the delay will be minimal $\left(\gtrsim 10^{7}\right.$ years), in good agreement with Maio et al. (2011). In their work, they also find similar reductions in halo gas fractions of up to a factor of two, even given their larger box size and lower resolution. This is furthermore consistent with other recent work such as that of Tseliakhovich et al. (2010).

The effect on reionization should be similarly minimal. Though not yet known with certainty, recent work has suggested that the sources of reionization were dominated by early galaxies of virial temperatures above the hydrogen cooling threshold of $10^{4} \mathrm{~K}$ (corresponding to masses $\gtrsim 10^{8} M_{\odot}$ ), with a much smaller contribution from $\lesssim 10^{6} M_{\odot}$ halos (e.g., Trac \& Gnedin 2009; Trenti \& Stiavelli 2009; Muñoz \& Loeb 2010). The relative streaming motions will do little to alter the infall of gas into the larger potential wells of ionizing galaxies, and reionization should proceed virtually unaffected. The effect of streaming is 
most pronounced at the highest redshifts when the collapse fraction and the corresponding radiative effects of stars are exceedingly small.

In conclusion, we have directly simulated the delay in collapse and the subsequent thermal evolution of the first baryonic structures under relative bulk velocities between gas and DM. Our results show that early star formation and subsequent evolution of reionization should quickly converge to the no-streaming case. Thus, results of previous and future cosmological studies concerning the formation of the first stars and galaxies will need only minimal modifications due to the relative streaming effect.

V.B. acknowledges support from NSF grants AST0708795 and AST-1009928, as well as NASA ATFP grants NNX08AL43G and NNX09AJ33G. The simulations were carried out at the Texas Advanced Computing Center (TACC).

\section{REFERENCES}

Barkana, R., \& Loeb, A. 2001, Phys. Rep., 349, 125

Bate, M. R., \& Burkert, A. 1997, MNRAS, 288, 1060

Bromm, V., Coppi, P. S., \& Larson, R. B. 2002, ApJ, 564, 23
Bromm, V., \& Larson, R. B. 2004, ARA\&A, 42, 79

Bromm, V., \& Loeb, A. 2004, New Astron., 9, 353

Bromm, V., Yoshida, N., Hernquist, L., \& McKee, C. F. 2009, Nature, 459, 49

Ciardi, B., \& Ferrara, A. 2005, Space Sci. Rev., 116, 625

Dalal, N., Pen, U., \& Seljak, U. 2010, J. Cosmol. Astropart. Phys., JCAP11(2010)007

Eisenstein, D. J., \& Hut, P. 1998, ApJ, 498, 137

Glover, S. 2005, Space Sci. Rev., 117, 445

Greif, T. H., Glover, S. C. O., Bromm, V., \& Klessen, R. S. 2010, ApJ, 716, 510

Haiman, Z., Thoul, A. A., \& Loeb, A. 1996, ApJ, 464, 523

Loeb, A. 2010, How Did the First Stars and Galaxies Form? (Princeton: Princeton Univ. Press)

Maio, U., Koopmans, L. V. E., \& Ciardi, B. 2011, MNRAS, 197 (arXiv:1011.4006)

Muñoz, J. A., \& Loeb, A. 2010, ApJ, submitted (arXiv:1010.2260)

Springel, V., \& Hernquist, L. 2002, MNRAS, 333, 649

Springel, V., Yoshida, N., \& White, S. D. M. 2001, New Astron., 6, 79

Tegmark, M., Silk, J., Rees, M. J., Blanchard, A., Abel, T., \& Palla, F. 1997, ApJ, 474,1

Trac, H., \& Gnedin, N. Y. 2009, arXiv:0906.4348

Trenti, M., \& Stiavelli, M. 2009, ApJ, 694, 879

Tseliakhovich, D., Barkana, R., \& Hirata, C. 2010, arXiv:1012.2574

Tseliakhovich, D., \& Hirata, C. 2010, Phys. Rev. D, 82, 083520

Yoshida, N., Abel, T., Hernquist, L., \& Sugiyama, N. 2003, ApJ, 592, 645

Yoshida, N., Omukai, K., Hernquist, L., \& Abel, T. 2006, ApJ, 652, 6

Zeldovich, Y. B. 1970, A\&A, 5, 84 